\title{
Riscos do uso indiscriminado de AINES em idosos
}

\author{
Risks of indiscriminate use of NSAIDs in the elderly
}

\author{
Alex Carioca De Oliveira Junior ${ }^{1 *}$, Lidiane Andressa Cavalcante Uhlmann ${ }^{\circ}$
}

Faculdade de Palmas, Palmas, Tocantins, Brasil.*Autor para correspondência. E-mal: alexjcdo@gmail.com

\begin{abstract}
Resumo: Introdução: Os anti-inflamatórios não esteroidais (AINES), estão classificados entre os medicamentos mais prescritos e comercializados em todo mundo. São empregados na terapia de dores provenientes de processo inflamatório. $\mathrm{O}$ crescimento no número de enfermidades crônicas em pessoas de idade avançada é considerado uma das principais comorbidades do processo de envelhecimento, em função dessa decorrência o consumo de medicamentos também houve um aumento nessa faixa de idade. Um estudo realizado em várias cidades brasileiras demonstra que em média 70-90\% nessa faixa etária fazem o uso contínuo de medicamentos, com uma média de dois a cinco medicamentos por pessoa. Revisão: O presente estudo trata-se de um levantamento bibliográfico de natureza exploratória, que tem como base o levantamento de dados científicos secundários e a sistematização das informações. A partir de bancos de dados como SCIELO, MEDLINE, PUBMED, PUBSAUDE, Manuais do Ministério da Saúde, tendo como data de corte os últimos 20 anos (2000-2020). Foram considerados aptos estudos publicados em espanhol, português e inglês. Estudos que não tratam do assunto, sobre idosos, automedicação ou anti-inflamatórios, estudos em outras línguas, ou anteriores a data de corte foram excluídos da pesquisa. Discussão: Os AINES devem ser prescritos com mais cautela e orientação específica para cada individualidade dos pacientes, especificamente os de idade mais avançada. Mesmo diante de toda sua qualidade e eficácia no tratamento de dores e inflamação das doenças crônicas. A venda dessa classe de medicamentos necessita ser mais restritas, e o fácil acesso a essa classe nas drogarias contribuem para uso indiscriminado e ao agravamento do quadro de saúde dos idosos, que já possuem alguma enfermidade em seu diagnóstico clínico, contribuindo para possíveis interações medicamentosas. considerações finais: Os anti-inflamatórios não esteroidais podem causar diversos riscos à saúde do paciente se não usado corretamente, sendo necessário uma atenção redobrada na dispensação e orientação ao paciente idoso sobre a maneira apropriada de uso do medicamento e possíveis intervenções com outros métodos para tratar suas enfermidades.
\end{abstract}

Palavras-chave: anti-inflamatórios, idosos, uso indiscriminado, riscos para saúde, reações adversas.

Abstract: Introduction: Non-steroidal anti-inflammatory drugs (NSAIDs) are among the most prescribed and marketed drugs worldwide. The increase in the number of chronic diseases in the elderly is considered one of the main diseases of the aging process, and because of this the consumption of medicines has also increased in this age group. A study conducted in several Brazilian cities shows that an average of $70-90 \%$ in this age group use medications continuously, with an average of two to five medications per person. Review: The present study is a bibliographical survey of an exploratory nature, based on the survey of secondary scientific data and the systematization of information. From databases such as SCIELO, MEDLINE, PUBMED, PUBSAUDE, Manuals of the Ministry of Health, using as cut-off date the last 20 years (2000-2020). Studies published in Spanish, Portuguese and English were considered suitable. Studies not dealing with the subject, about the elderly, self-medication or anti-inflammatory drugs, studies in other languages, or studies prior to the cut-off date were excluded from the search. Discussion: NSAIDs should be prescribed with more caution and specific orientation for each individual patient, specifically the older ones. Even with all its quality and effectiveness in the treatment of pain and inflammation of chronic diseases. The sale of this class of drugs needs to be more restricted, and the easy access to this class in drugstores contributes to indiscriminate use and the worsening of the general health condition of the elderly, who already have some disease in their clinical diagnosis, contributing to possible drug interactions. Final considerations: Nonsteroidal anti-inflammatory drugs can cause several risks to the patient's health if not used correctly. It is necessary to pay special attention to the dispensation and orientation of the elderly patient on the correct use of the drug and possible interventions with other methods to treat their diseases

Keywords: anti-inflammatorium, idoses, indiscriminated use, health risks, adverse reactions. 


\section{Introdução}

O aumento na expectativa do tempo de vida é um dos grandes feitos na sociedade, com a acrescente na saúde humana, bem como novos métodos na medicina e tratamentos preventivos, mesmo que em diferentes escalas de acordo com países mais desenvolvidos economicamente. Chegar à velhice com uma boa saúde, já é possível, também em países mais pobres (Closs \& Schwnake, 2012).

Segundo o Estatuto do idoso (Brasil, 2003), toda pessoa idosa tem direito à saúde, trabalho, educação, cultura, esporte, habitação e meio de transporte. Projeções populacionais feitas pelo Instituto Brasileiro de Geografia e Estatística, mostram que até 2060 cerca de $25,5 \%$ da população brasileira tenha 60 anos ou mais (IBGE, 2009).

Esse considerável aumento na população também pode se tornar um grande desafio daqui em diante, podendo faltar mão de obra com idade trabalhadora, já os idosos usufruem de mais ofícios de saúde, as internações hospitalares habituam ser mais frequentes e a ocupação do leito é maior comparado a outras idades. Sendo necessário um cuidado mais minucioso, acompanhamento continuo e exames periódicos (Ramos et al., 2008).

O processo de envelhecimento provoca alterações no corpo tais como problemas cardiovasculares, respiratórias, na pele, no sistema digestivo, e muscular (Monteiro, 2001).

$\mathrm{O}$ aumento de doenças crônicas em idosos é considerado uma das principais enfermidades do processo de envelhecimento. Dados indicam que essas doenças correspondem em média de $66,3 \%$ das patologias acometidas em idosos (Campolina et al., 2013). Em função dessa decorrência o consumo de medicamentos também aumenta nessa faixa de idade (Silva et al., 2012).

Um estudo de Coelho et al. (2004), realizado em várias cidades brasileiras demonstram que em média 7090\% nessa faixa etária fazem o uso contínuo de medicamentos, com média de dois a cinco medicamentos por pessoa.

A polimedicação pode ter o benefício de tratar várias enfermidades ao mesmo tempo, entretanto também eleva o risco de reações adversas e interações medicamentosas e torna a assistência farmacêutica mais difícil. Relacionado diretamente à sintomas de dores, as doenças crônicas influenciam aos idosos tomarem analgésicos e anti-inflamatórios por conta própria, fazendo a automedicação (Cascares et al., 2008).

Os anti-inflamatórios não esteroidais (AINES) estão entre os medicamentos mais prescritos e comercializados em todo mundo. Utilizados no tratamento de dores crônicas e agudas provenientes de processo inflamatório. São considerados como analgésicos antipiréticos e agem diretamente na inflamação, essas três indicações são de grande uso na pratica clínica (Howard, 2004).

Com o que foi demonstrado, este trabalho tem como objetivo enumerar os riscos causados pelos AINES em idosos se usados de forma indiscriminada e sem a orientação correta

\section{Revisão}

O presente estudo trata-se de uma revisão de literatura e levantamento bibliográfico. O estudo teve como base o levantamento de dados científicos secundários e a sistematização das informações. A partir de bancos de dados em saúde, como, Scientific Electronic Library Online (SCIELO), MEDLINE, PUBMED, PUBSAÚDE, Manuais do Ministério da Saúde.

A pesquisa foi realizada com as seguintes palavras chaves: "Anti-inflamatórios", "Idosos", "Uso indiscriminado", "revisão de literatura", "reações adversas". Foram considerados selecionáveis estudos publicados em espanhol, português e espanhol, disponíveis em revistas eletrônicas nos últimos 20 anos (2000-2020). Estudos que não tratam do assunto, sobre idosos, automedicação ou anti-inflamatórios, estudos em outras línguas, ou anteriores a data de corte foram excluídos da pesquisa.

O processo de realização desse artigo ocorreu em analisar o título e resumo e data de publicação, e se enquadrasse nos parâmetros foi lido o texto geral. Atendendo os critérios foi feito o resumo e descrito as partes com mais relevância.

Tratando-se de uma revisão de literatura, não foi necessária uma aprovação do comitê de ética e pesquisa.

\section{Discussão}

Indicados para o alívio da dor e inflamação, os anti-inflamatórios não esteroidais estão entre os mais utilizados para o tratamento em artrite reumatóide, osteoartrite e dores comuns como dores de cabeça ou tendinites. A dor envolve vários componentes que podem ser tratados de outra forma com meios não 
farmacológicos, utilizando outras áreas da medicina, porém, o tratamento farmacológico ainda tem o meio de acesso mais simplificado para qualquer cidadão (Mainar et al., 2009).

A isoenzima COX-1 é expressa de forma estável na maior parte dos tecidos; a isoenzima COX-2 é induzida diretamente nos processos inflamatórios. A COX-1 é de suma importância para a sustentação do estado fisiológico natural de vários tecidos, abrangendo a proteção da mucosa do trato gastrointestinal, controle do fluxo sanguíneo renal, respostas autoimunes, e do sistema nervoso central, cardiovasculares e reprodutivas (Antman et al., 2005).

A COX-2, induzida diretamente no processo inflamatório por vários estímulos, como; citocinas, endotoxinas e fatores de crescimento, da origem à prostaglandinas induzidoras, que contribuem no processo do edema, rubor, febre e dor. A COX-2 se expressa também nas células vasculares endoteliais normais, que secretam prostaciclina em resposta a contusão ou lesão (Fitzgerald, 2004).

O mecanismo de ação destes medicamentos está relacionado com a inibição periférica e central da atividade das enzimas ciclo-oxigenase (COX-1 e COX-2) e subsequente diminuição da biossíntese e liberação dos mediadores da inflamação, dor e febre, as prostaglandinas (Silva, 2002).

Os AINES não seletivos da COX inibem a produção de prostaglandinas na mucosa gastrointestinal, podendo causar úlcera gástrica e sangramento digestivo. Esses AINES reduzem a produção plaquetária do tromboxano A2 (TXA2), devido ao bloqueio da COX-1, e previnem a trombose arterial. Recentemente, tem sido indicado que os inibidores seletivos da COX-2 aumentam o risco cardiovascular. Esses agentes não bloqueiam a formação de TXA2, nem exercem ação antiplaquetária, devido à inibição mínima da COX-1, porém reduzem a produção de prostaciclina (Gerald \& Patrono, 2001).

Segundo Silva (2002), os AINES inibindo as ciclooxigenases, podem provocar vários efeitos colaterais em idosos como: hemorragia gastrointestinal, diarréia, úlcera péptica, dispepsia, disfunção e falência renal, inibição da agregação plaquetária consequentemente o aumento do tempo de sangramento, alterações das funções renais.

Atualmente eles são classificados em seletivos da COX-1, os não-seletivos, seletivos da COX-2 e altamente seletivos da COX-2 (Wagner et al., 2006). Os principais AINES dentre os mais utilizados estão listados no quadro 1.

Quadro 1. classificação dos AINES quanto a seletividade.

\begin{tabular}{|c|c|c|c|}
\hline $\begin{array}{c}\text { Inibidores seletivos da COX- } \\
\mathbf{1}\end{array}$ & $\begin{array}{c}\text { Inibidores não seletivos da } \\
\text { COX }\end{array}$ & $\begin{array}{c}\text { Inibidores seletivos da } \\
\text { COX-2 }\end{array}$ & $\begin{array}{c}\text { Inibidores altamente seletivos para } \\
\text { COX-2 }\end{array}$ \\
\hline $\begin{array}{c}\text { Ácido acetilsalicílico (baixas } \\
\text { doses) }\end{array}$ & $\begin{array}{c}\text { Ácido acetilsalicílico (altas } \\
\text { doses) }\end{array}$ & Nimesulida & Celecoxibe \\
\hline & Piroxicam & Meloxicam & Etoricoxibe \\
\hline & Diclofenaco & Etodolaco & Paracoxibe \\
\hline & Ibuprofeno & & Lumiracoxibe \\
\hline
\end{tabular}

Fonte: Monteiro et al. (2008).

De acordo com O'Neil et al. (2012), determinados estudos americanos aconselham que os antiinflamatórios orais devem ser cuidadosamente prescritos ou administrados com muita precaução para o tratamento de dor e inflamação em pacientes idosos. O uso de AINES em idosos está relacionado ao elevado risco de toxicidade gastrointestinal e insuficiência renal, além de estar diretamente ligado a eventos cardiovasculares e a interações medicamentosas.

Um estudo feito na cidade de Porto Alegre no Rio Grande do Sul por Ely et al. (2015), analisou o uso dos anti-inflamatórios na estratégia da saúde e da família da cidade e teve a conclusão que quanto aos aspectos sócio demográficos, o uso de anti-inflamatórios e analgésicos era feito especialmente pelas idosas.

$\mathrm{O}$ uso de medicamentos pelas idosas está descrito em vários estudos, sendo que as mulheres normalmente tem maior preocupação com a sua saúde e procuram mais os cuidar da saúde do que os homens (Flores \& Benvegnu, 2008).

\section{Efeitos no estômago}

Grande parte dos pacientes idosos que fazem o tratamento com algum tipo de anti-inflamatório não terminam o tratamento completo devido aos efeitos adversos no trato gastrointestinal como dor abdominal, azia e diarréia, o tratamento em longo prazo pode causar ulceras gástricas e duodenais (Vakil, 2020).

As decorrências do bloqueio da COX-1 realizada pelos AINES no trato gastrointestinal (TGI) são; a inibição da proteção da mucosa nas prostaciclinas, PGE2 e PGD2 e o aumento da secreção do ácido 
clorídrico, causando uma possível ulceração, perfuração e hemorragia. A inibição da síntese de PGs, causa no estômago uma maior vulnerabilidade às lesões, o que levou o nome de gastropatia por AINES. Os efeitos gastrintestinais provocados pelo uso prolongado podem ser leves, como dispepsia e ulceras gastrintestinais, até alcançar níveis mais sérios, como anemia e sangramento gastrintestinal grave. Causando problemas graves ao paciente idoso (Wagner et al., 2006).

As chances de ocorrer úlcera ou sangramento aumentam com o uso em dosagens altas ou prorrogadas dos AINES, o uso concomitante de corticosteróides ou anticoagulantes, bebidas alcoólicas e idade avançada. Para diminuir esses eventos em pacientes com ulceras, gastrites ou sangramentos no TGI, devem usar os medicamentos da classe inibidores da bomba de prótons em jejum diariamente e fazer a administração dos AINES após a refeição. Devido à grande incidência de eventos adversos no trato gastrointestinal, a partir de 1999 foram desenvolvidos e introduzidos no mercado os inibidores seletivos da COX-2, chamados de COXIBEs com o objetivo de minimizar esses efeitos adversos (Abraham et al., 2007).

\section{Efeitos cardiovasculares}

Os COXIBEs são equivalentes ou até mais eficazes que os AINES para o tratamento de dores e inflamações, entretanto eles não têm atividade antitrombótica, pois as plaquetas expressam a COX-1. Um primeiro estudo feito por VIGOR, (2000), com o rofecoxibe, que já foi descontinuado do mercado, demonstrou o comparativo entre o tratamento de rofecoxibe 50mg/dia com o naproxeno $500 \mathrm{mg}$ duas vezes ao dia, observou-se uma maior incidência de infarto agudo do miocárdio com o uso do rofecoxibe $(0,4 \%$ ao ano) já o naproxeno $(0,1 \%$ ao ano).

Essa disposição a trombose em relação ao coração dá-se pelo desvio do equilíbrio pro trombótico e antitrombótico na superfície endotelial, aumentando o risco de isquemias e infarto agudo do miocárdio principalmente em pacientes idosos. Em conclusão, o aumento significativo de eventos cardiovasculares está associado com os inibidores seletivos da COX-2 (Abraham et al., 2007).

Um estudo realizado por Garcia et al. (2008), avaliaram sobre a associação entre dose, frequência e duração de diferentes AINES e o risco de infarto agudo do miocárdio em diferentes idades. Foram identificados 8852 casos de infartos não fatais em pacientes idosos de 49 a 80 anos, entre 2000 e 2005 . Registrou-se o risco de infarto com o grau de inibição da COX-1 e COX-2.

O uso concomitante de alguns AINES, como o ibuprofeno, diclofenaco e o rofecoxibe, com o ácido acetilsalicílico (AAS) interferem na capacidade de inibir irreversivelmente a COX-1, não fazendo o efeito de bloquear a agregação plaquetária, logo não protegendo contra eventos aterotrombóticos (Lana \& Sheiman, 2007).

\section{Aumento da pressão arterial}

O poder de inibição das enzimas COX-1 e COX-2 apresentado pelos AINES é o principal mecanismo que afeta a elevação da pressão arterial, em pacientes de todas as idades principalmente idosos, que gera a redução sistêmica e renal da síntese de PGs. Desse modo, todos os AINES podem diminuir a ação dos antihipertensivos, seja parcial ou total. Outros medicamentos com ação analgésica e antitérmica, como a dipirona e o paracetamol, também podem intervir na ação dos anti-hipertensivos (Silva et al., 2008).

De acordo com Fortes \& Nigro (2005), as PGs são de extrema importância no processo de resposta inflamatória, além de serem importantes fisiologicamente na modulação da dilatação vascular renal e sistêmica, na secreção tubular de sódio e água, na transmissão adrenérgica, no sistema reninaangiotensina- aldosterona e na proteção da mucosa gástrica.

Um estudo de Mota et al. (2010), verificou a utilização de anti-inflamatórios não esteroidais prescritos para idosos da região de São Paulo e expuseram que em quatro prescrições houve a administração conjunta de algum AINE e um anti-hipertensivo. Com isso, descreveram que esta associação pode causar a diminuição do efeito de diminuir a pressão arterial em pacientes hipertensos, logo que o enalapril (IECA) e o atenolol (betabloqueador) necessitam das prostaglandinas renais para cumprirem seu mecanismo de ação, e quando há uso conjunto de um AINE, ocorre à diminuição da ação por causa da inibição da síntese de PGs.

Diversos dados também mostraram que os AINES interferem com os anti-hipertensivos das diversas classes desses medicamentos, especialmente daquelas no qual o mecanismo de ação envolve também a síntese das prostaglandinas. Os bloqueadores dos canais de cálcio e antagonistas dos receptores de angiotensina II sofreram menor interferência dos AINES em seus efeitos (Rodríguez et al., 2008).

\section{Efeitos renais}

Assim como o efeito sobre a pressão arterial, o efeito sobre a função renal está diretamente relacionado à diminuição da síntese de PG induzida pelos AINES. As PGs são importantes reguladores fisiológicos do 
tônus vascular renal e do equilíbrio hídrico, e estão envolvidos na reabsorção de sódio e água pelos túbulos renais. Além disso, o PG funciona protegendo o fluxo sanguíneo renal e a filtração glomerular. Aproximadamente $25 \%$ dos pacientes apresentarão retenção de sódio, o que leva a um aumento na quantidade de líquido extracelular e na pressão arterial. Pacientes que já têm pressão alta aumentam a pressão de forma mais significativa (Michelin et al., 2006).

Danos renais causados por anti-inflamatórios não esteróides podem causar nefrite aguda e síndrome nefrótica, que pode ser encontrada em muitos pacientes que tomam esses AINES. Essas complicações renais são reversíveis após a interrupção do tratamento medicamentoso. No entanto, em condições adversas, pessoas que já têm doença renal, podem causar disfunção renal aguda e necrose papilar renal (Monteiro et al., 2008).

\section{Considerações finais}

Portanto, sabendo de toda sua qualidade e eficácia no tratamento de dores e inflamação das doenças crônicas, os AINES devem ser prescritos com mais cautela e orientação especifica para cada individualidade dos pacientes, especificamente os de idade mais avançada. A venda dessa classe de medicamentos necessita ser mais restritas, e o fácil acesso a essa classe nas drogarias contribuem para uso indiscriminado e ao agravamento do quadro geral de saúde dos idosos, que já possuem alguma enfermidade em seu diagnostico clinico, contribuindo para possíveis interações medicamentosas.

Pelos relatos e análises das pesquisas, vemos que os AINES são amplamente utilizados como primeira escolha para dor e inflamação em várias faixas etárias, principalmente em idosos, porém, de acordo com esta revisão, entende-se que ainda falta esclarecimento e ajuda ao idoso e a informação da população sobre o uso simultâneo dos diversos medicamentos citados neste trabalho, essas informações podem ser aprimoradas por meio da atenção farmacêutica. Os profissionais de saúde devem estar comprometidos com o uso racional de medicamentos, pois o índice de uso inadequado de medicamentos por automedicação é elevado. Também é muito importante a realização de estudos frequentes de interação medicamentosa para profissionais de saúde, a fim de reduzir as prescrições de medicamentos incorretas e melhorar a qualidade de vida dos pacientes.

\section{Referências}

Abraham, N., El-serag H. B., Hartman, C., Richardson, P., \& Deswal, A. 2007. Cyclooxygenase-2 selectivity of non-steroidal anti-inflammatory drugs and the risk of myocardial infarction and cerebro vascular accident. Alimentary Phamarcolyand Therapeutics, 25, 913-24.

Antman, E. M., DeMets D., \& Loscalzo J. 2005. Cyclooxygenase inibitionand cardiovascular risk. Circulation, $112,759-70$.

Batlouni, M. 2010. Anti-inflamatórios não esteroides: Efeitos cardiovasculares, cérebro - vasculares e renais. Arquivo Brasileiro de Cardiologia, 94, 556-63

Bombardier, C. M. D., Laine L., Reicin, A., Shapiro, D., Vargas, R. B., Davis, B., Day, R., Ferraz, M. B., Hawkey, C. J., Hochberg, M. C., Kevien, T. K., Schnitzer, T. J. for the VIGOR Study Groupe. 2000. Comparison of upper gastrointestinal toxicity of rofecoxib and naproxen in patients with rheumatoid arthritis. New England Journal of Medicine, 343, 1520-8.

Brasil, Lei $\mathrm{n}^{\mathrm{o}}$ 10.741, de 01 de outubro de 2003. 2003. Dispõe sobre o Estatuto do Idoso e dá outras providências. Brasília, DF: Diário Oficial da União.

Campolina, A. G., Adami F., Santos J. L. F., \& Lebrão M. L. 2013. A transição de saúde e as mudanças na expectativa de vida saudável da população idosa: possíveis impactos da prevenção de doenças crônicas. Caderno de Saúde Pública, 29(6),1217-29.

Cascaes, E. A., Falchetti, M. L., \& Galato, D. 2008. Perfil da automedicação em idosos participantes de grupos da terceira idade de uma cidade do sul do Brasil. Arquivo Catarinense de Medicina, 37(1),63-9.

Closs, V. E., \& Schwnake, C. H. A. 2012. A evolução do índice de envelhecimento no Brasil, nas suas regiões e unidades federativas no período de 1970 a 2010. Revista Brasileira de Geriatria e Gerontologia, 15(3), 443-458.

Coelho, F. J. M., Marmopito, L. F., \& Castelo, A. 2004. Perfil de utilização de medicamentos por idosos em área urbana do nordeste do Brasil. Revista de Saúde Pública, 38(4), 557-64. 
Ely, L. S., Engroff, P., Guiselli R. S., Cardoso, G. C., Morrone, F. B., \& Carli, G. A. 2015. Uso de antiinflamatórios e analgésicos por uma população de idosos atendida na Estratégia Saúde da Família. Revista Brasileira de Geriatria e Gerontologia, 18(3).

Fitzgerald, G. 2004. Coxib sand cardiovascular disease. New England Journal Medicine, 351,1709-11

Flores, V. B., \& Benvegnú, L. A. 2008. Perfil de utilização de medicamentos em idosos da zona urbana de Santa Rosa, Rio Grande do Sul, Brasil. Caderno de Saúde Pública, 24(6),1439-46.

Fortes, Z. B., \& Nigro, D. 2005. Aspectos farmacológicos da interação anti-hipertensivos e antiinflamatórios não esteroides. Revista Brasileira do Hipertenso, 12(2), 108-111.

Gerald, G. A., \& Patrono, C. 2001. The coxibs, selective inhibitors of cyclooxygenase-2. England Journal of Medicine, 345, 433-42.

Howard, P. A. 2004. Nonsteroidal anti-inflamatory drugs and cardiovascular Risk. Journal American Coll Cardiology, 43, 519-25.

Instituto Brasileiro de Geografia e Estatística [IBGE]. 2009. Estudos e Pesquisas. Informação Demográfica e Socioeconômica, número 25. Indicadores Sociodemográficos e de Saúde no Brasil. A Dinâmica Demográfica Brasileira e os Impactos nas Políticas Públicas. Rio de Janeiro, RJ: IBGE.

Kummer, C. L., \& Coelho T. C. R. B. 2002. Anti-inflamatórios Não Esteroides Inibidores da Ciclooxigenase-2 (COX-2): Aspectos Atuais. Revista Brasileira de Anestesiologia, 52,498-512.

Lanas, A., \& Scheiman, J. 2007. Low-dose aspirin and upper gastrointestinal damage: epidemiology, prevention and treatment. Current Medical ResearchOpinion, 23, 163-73.

Mainar, A., Florensa, S., \& Artieda, R. N. 2009. Consumption of oral analgesics and dosage forms in elderly patients: population based study. Farmacia hospitalaria, 33(3),161-171.

Michelin, F., Ferreira, A. A. P., Galvani, B., \& Cruz, L. 2006. Toxicidade Renal de Inibidores Seletivos da Cicloxigenase-2: Celecoxibe e Rofecoxibe. Revista Científica Médica, 321-332.

Monteiro, P. P. 2001. Envelhecer: histórias, encontros, transformações. Belo Horizonte, MG: Autêntica.

Monteiro, E, C. A., Trindade, J. M. F., Duarte, A. L. B. P., \& Chahade, W. H. 2008. Os Anti-inflamatórios não esteroidais (AINES). Temas de Reumatologia Clínica, 9(2): 53-63.

Mota, P. M., Lima, A. L. Z., Coelho, E., Paula, E. M. X., \& Furine, A. A. C. 2010. Estudo sobre a utilização de anti-inflamatórios não esteroidais prescritos em receitas para idosos da região Noroeste Paulista. Revista Ciência Farmacêutica Básica, 31(2),157-163.

O'neil, C. K. O., Hanlon, J. T., \& Marcum, Z. A. 2012. Adverse effects of analgesics commonly Used by older adults with osteoarthritis: focus on non- opioid and opioid analgesics. American Journal Geriat. Pharmacot, 10(6),331-42.

Paradella, R. 2018. Instituto Brasileiro de Geografia e Estatística (IBGE), ed. Estatísticas sociais.

Ramos, E. L, Souza, N. V. D . O., \& Caldas, C. P. 2008. Qualidade de vida do idoso trabalhador. Ver. Enfermagem, 16(4):,507-11.

Rodríguez, L. A. G., Tacconelli, S., \& Patrignani, P. 2008. Role of dose pontency in the prediction of risk of myocardial infarction associated with nosnteroidal anti-inflammatory drugs in the general population. Journals of the American College of Cardiology, 52,1628-1636.

Silva, J. E. D., Sette, I. M. F., Belem, L. F., Pereira, G. J. S., \& Barbosa, J. A. A. 2008. Interação medicamentosa entre anti-inflamatórios não esteroides e anti-hipertensivo em pacientes hipertensos internados em um hospital público: uma abordagem em farmacovigilância. Revista Baiana de Saúde Pública, 32(1):, 18-28.

Silva, P. 2002. Farmacologia (6a ed.). Rio de Janeiro, RJ: Guanabara Koogan.

Silva A. L., Ribeiro, A. Q., Clein, C., Acurcio F. 2012. Utilização de medicamentos por idosos brasileiros, de acordo com a faixa etária: um inquérito postal. Caderno de Saúde Pública, 28(6), 1033-1045.

Vakil N. 2020. Ulcerous peptic. University of Wisconsin School of Medicine and Public Health.

Wagner, W., Kanna, P., Furst, D. 2006. Anti-inflamatórios não esteroides, Fármacos Antirreumáticos Modificadores da Doença, Analgésicos não opioides e Fármacos Utilizados na Gota. In KATZUNG, B.G. (Edit). Farmacologia Básica e Clínica (9a ed.). Rio de Janeiro, RJ: Guanabara Koogan.

\section{Minicurrículo}

Alex Carioca De Oliveira Junior.Bacharelando em Farmácia, cursando o último ano de Farmácia pela Faculdade de Palmas - FAPAL.Atualmente há 8 anos no mercado farmacêutico 
Lidiane Andressa Cavalcante Uhlmann.Mestre em Biodiversidade, Ecologia e Conservação - PPGBEC UFT (2019) e Licenciada em Ciências Biológicas pela Universidade Federal do Tocantins (2016). Docentena Faculdade de Palmas - FAPAL, desde 2019. link currículo lattes - http://lattes.cnpq.br/5589706899405212

Como citar: Oliveira Junior, A.C., \& Uhlmann, L.A.C. 2021. Riscos do uso indiscriminado de AINES em idosos ,6, 154. DOI: https://dx.doi.org/10.31533/pubsaude6.a202

Recebido: 25 mai. 2021.

Revisado e aceito: 7 jun. 2021.

Conflito de interesse: os autores declaram, em relação aos produtos e companhias descritos nesse artigo, não ter interesses associativos, comerciais, de propriedade ou financeiros que representem conflito de interesse.

Licenciamento: Este artigo é publicado na modalidade Acesso Aberto sob a licença Creative Commons Atribuição 4.0 (CC-BY 4.0). 